\title{
Concavities count for less in symmetry perception
}

\author{
JOHAN Hulleman \\ University of Hull, Hull, England \\ AND \\ Christian N. L. Olivers \\ Vrije Universiteit, Amsterdam, The Netherlands
}

\begin{abstract}
We investigated the relative importance of convexities (protrusions) and concavities (indentations) for the perception of shape. On the one hand, it has been suggested that convexities determine the shape of an object, whereas concavities merely act as "perceptual glue" between the convexities. On the other hand, it has been argued that concavities are more salient than convexities. We show that participants find it easier to detect asymmetry in a 2-D silhouette when there is a mismatch between the shapes of convexities on either side of the axis of symmetry than when there is a mismatch between the shapes of concavities. This is the case even when the concavities are closest to the axis of symmetry, and despite the usual bias toward this axis in symmetry perception. We suggest that the actual shape of concavities is less important in symmetry perception, because the main role of concavities is to act as part boundaries in the representation of the shape of objects.
\end{abstract}

One of the most easily observed shape properties of 2-D patterns is bilateral symmetry (see Wagemans, 1995, for a review). As first argued by Julesz (1971), the area around the axis of symmetry is the most important. He found that the symmetry of a small strip around the axis determined whether a random dot texture was perceived as symmetrical or not, regardless of the properties of areas farther away from the axis. However, as pointed out by Wagemans (1995), for the percept of symmetry in 2-D shapes rather than dot patterns, areas farther away from the symmetry axis must contribute too. For instance, the participants in Palmer and Hemenway's (1978) study had to detect symmetry in briefly presented outline shapes without any internal features close to the axis of symmetry, and could do so well.

Nevertheless, even in symmetrical 2-D shapes, some regions are typically closer to the axis than others. This is often the case for concave areas of the shape, whereas convex areas are usually farther away. By their very nature, convexities are protruding, taking the contour away from the axis, whereas concavities are indentations, taking the contour closer to the axis. The question, then, is whether the same bias toward the axis exists for symmetry perception in 2-D shapes as it does for texture patterns. In other words, will shape properties of concave regions close to the axis be most important for the percept of symmetry, or is symmetry perception dominated by the convex regions farther away from the axis? Different theories seem to diverge on this.

According to some accounts, symmetry perception is a fast, parallel, preattentive process (e.g., Baylis \& Driver,
1994; Julesz, 1971; see Wagemans, 1995, for a review) that compares low-level visual features across the axis (with a notable bias toward the vertical axis). Such comparisons are facilitated by the relative proximity of the to-be-compared features to each other, and hence, to the axis. On the assumption that such fast feature comparisons could occur before shape perception, we should expect the aforementioned bias toward the axis.

However, others have argued that symmetry perception is governed by shape perception. Evidence has been provided by Baylis and Driver (1995), who argued that symmetry is only processed after shape perception-that is, after convexities and concavities have been coded for. They found that symmetry perception is speeded when both sides of a symmetrical silhouette have the same sequence of concavities and convexities. If, because of figure-ground assignment, symmetrical curves receive different shape descriptions (i.e., convex, on one curve, paired with concave, on the other) symmetry perception is impeded. Hence, Baylis and Driver (1995) argued that figure-ground assignment has to precede symmetry perception. Also, Hulleman and Humphreys (2004) showed that symmetry perception is sensitive to the assignment of top and bottom to shapes, which again suggests that symmetry perception is affected by shape descriptions. Furthermore, Hulleman, Te Winkel, and Boselie (2000) have shown that concavities can be easily detected in visual search displays, whereas Olivers and Van der Helm (1998) found that this is not the case for bilateral symmetry. This also suggests that the representation of figural properties such as concavities is more fundamental than the repre-

J. Hulleman, j.hulleman@hull.ac.uk 
sentation of symmetry. Thus, according to this strand in the literature, the relative contribution of convexities and concavities in symmetry perception would be predicted to depend on their relative role in shape perception.

However, evidence on the relative importance of convexities and concavities from the shape perception literature is rather mixed. Several authors have emphasized the importance of convexities. For instance, Koenderink and Van Doorn (1982) have argued that throughout art history, convex regions have been considered to be plastic (i.e., to have shape), whereas the regions in between (concave and saddle-shaped regions) have been treated as "regions of transition - the glue that keeps things together but is of itself of little interest" (p. 131). Hoffman and Singh (1997) have also argued that convexities are the most salient. On the other hand, there are several lines of research that suggest that concavities are more salient. As mentioned earlier, Hulleman et al. (2000) reported easy detection of concavities in visual search displays (relative to convexities). More recently, Barenholtz, Cohen, Feldman, and Singh (2003) also found that the introduction of a concavity in a shape was more salient than the introduction of a convexity.

Such different strands of evidence are not necessarily in conflict. Hoffman and Richards (1984) identified concavities in 2-D shapes (more precisely, the minimum of negative curvature within the concavity) as the place where the contour of a silhouette might be broken up, so that the original constituting parts of the shape can be retrieved. So it might be that convexities and concavities are salient for different reasons: Concavities are salient because they are part boundaries, whereas convexities are salient because they are the (shaped) parts. Depending on the type of task (stressing part shapes or part boundaries), either convexities or concavities may then turn out to be more prominent. Here, we ask the question, which of the two is most prominent in symmetry perception?

We performed three experiments to address the question of whether or not concavities and convexities are equally important in the perception of symmetry. First, in Experi- ment 1, we used dot patterns to establish the typical bias toward the symmetry axis. The same type of dot patterns was then used as a basis to fit contours and create the 2-D silhouettes for Experiments 2 and 3. Those experiments then tested whether concavities and convexities differed in their importance for symmetry perception, given the bias found in Experiment 1. In Experiment 2, the concavities were closest to the axis of symmetry, whereas in Experiment 3, they were farthest away.

On the basis of low-level feature accounts of symmetry perception, we would expect a bias toward the axis in both Experiments 2 and 3 (given that such a bias would occur in Experiment 1). On the basis of shape perception accounts, predictions may go either way. In this sense, symmetry detection creates a perfect opportunity to compare convexities and concavities, because the shapes of both have to match for a silhouette to be symmetric.

\section{METHOD}

\section{Participants}

Twelve students of the Vrije Universiteit Amsterdam (5 male, all right-handed, ages 18 to 21 years, average age 19 years) participated in Experiment 1 . Twenty new students ( 11 male, 3 left-handed, ages 18 to 35 years, average age 21 years) participated in Experiment 2, and another 20 new students (11 male, 2 left-handed, ages 18 to 24 years, average age 21 years) participated in Experiment 3 . All of the students were reimbursed for their participation. None of them were aware of the purpose of the experiments.

\section{Stimuli}

Figure 1 illustrates the construction of the stimuli. The basic building blocks of all the stimuli were nine triads of points $\left(1.15^{\circ} \times\right.$ $1.15^{\circ}$ ), of which the tips were $0.15^{\circ}$ above, on, and $0.15^{\circ}$ below the horizontal midline of the triads (see Figure 1A). The triads could point leftward or rightward. To create a symmetrical stimulus, a vertical concatenation of seven randomly sampled (with replacement) triads was created (leftward and rightward pointing alternating; see Figure 1C), and flipped around the vertical (see Figure 1D). To create an asymmetrical stimulus, in one of the halves, the tips of either the top three leftward- or the top three rightward-pointing triangles were perturbed by moving them $0.25^{\circ}$ upward and $0.1^{\circ}$ leftward or

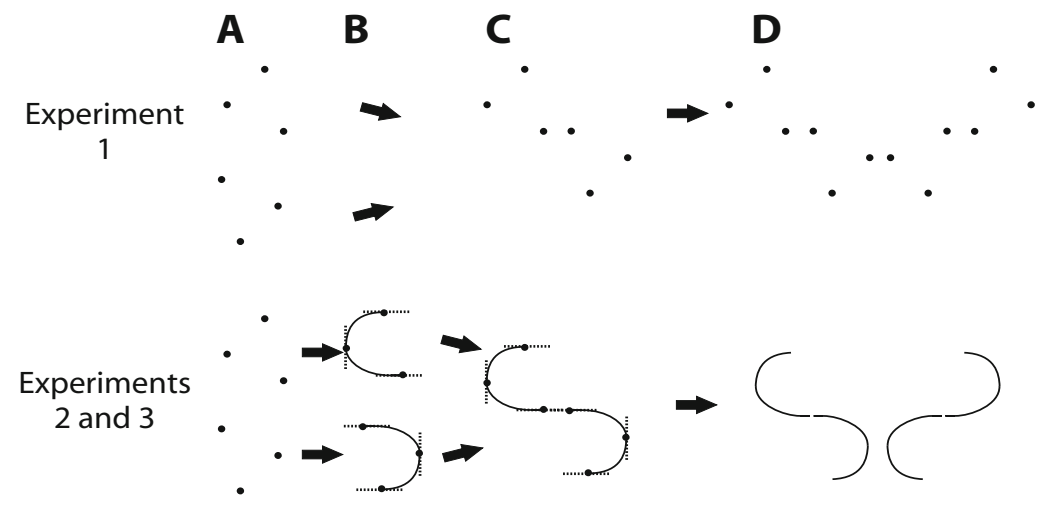

Figure 1. Stimulus creation: upper half, Experiment 1; lower half, Experiments 2 and 3. (A) Two triads of points. (B) To create the silhouettes used in Experiments 2 and 3, curves (Hermite polynomials) were fitted through each triad, under the constraint that the tangents were horizontal at the top and bottom points of the triad and vertical at the other point. (C) The triads are linked together. Because of the horizontal tangents for both top and bottom points of any triad, the link will be seamless. (D) Mirroring yields a symmetric stimulus. 
rightward. This way, families of 16 related stimuli (symmetrical and perturbed) could be created, and the experiments used 25 of such families each.

In Experiment 1, we presented only the points $\left(0.25^{\circ}\right.$ diameter $)$ of the triads (see Figure 2A). The entire dot pattern measured $5.3^{\circ} \times$ $8.0^{\circ}$. In Experiment 2, curves were fitted through the triads and the shape was filled, resulting in closed silhouettes (see Figure 1B for an illustration of this process; see Figure 2B for an example of the resulting stimulus). In Experiment 3, the same curves were fitted, but this time we created two closed silhouettes measuring $12.5^{\circ} \times$ $8.0^{\circ}$ in total (see Figure $2 \mathrm{C}$ ). As a result, what were concavities in Experiment 2 became convexities in Experiment 3, and vice versa. To ensure that participants saw two figures, we took several precautions. First, we closed the curves with a convex arc. Convexity is a strong figural cue (Palmer, 1999). Second, we added a texture gradient to the background. This texture gradient could be seen to continue behind the two figures. The gradient remained constantly visible throughout Experiment 3, creating the impression of a stable background with two figures presented on top of it. The brief presentation of the figures also contributed to this. Third, we used a new group of participants. This prevented the occurrence of any context effects, because the participants were never exposed to the stimuli used in Experiment 2. Admittedly, there may still be some residual figure-ground ambiguity in Experiment 3. Note, however, that we would expect Experiments 2 and 3 to generate the same pattern of results, if the figure-ground assignment would indeed be opposite to our intentions.

\section{Procedure and Design}

Stimulus presentation and response recording were done using E-Prime (Psychology Software Tools, Inc., Pittsburgh, PA). After a 1,000 -msec blank period, a $0.5^{\circ} \times 0.5^{\circ}$ fixation cross was presented for $500 \mathrm{msec}$ in the center of the display. $250 \mathrm{msec}$ after the offset of the fixation cross, the first stimulus was presented (for $200 \mathrm{msec}$ in Experiment 1, $100 \mathrm{msec}$ in Experiment 2, and $250 \mathrm{msec}$ in Experiment 3; presentation times were varied to keep overall levels of performance approximately equal across experiments and to prevent ceiling and floor effects). After a blank interval of $750 \mathrm{msec}$, the second stimulus was presented for the same duration. A display prompting the participants to reply appeared $250 \mathrm{msec}$ after the offset of the second stimulus, and remained visible until the participant had responded. The participant's task was to identify which of the two stimuli was symmetrical, by typing in either a " 1 " or a " 2 " on a standard U.S. keyboard. Participants were instructed to guess whenever they were uncertain. Each experiment started with 20 practice trials, followed by 10 blocks of 40 experimental trials, with a break after each block.

The only (within-subject) factor in each experiment was type of perturbation, with two levels for each experiment. These were near to axis and far from axis for Experiment 1; near to axis (concavities)
A Near

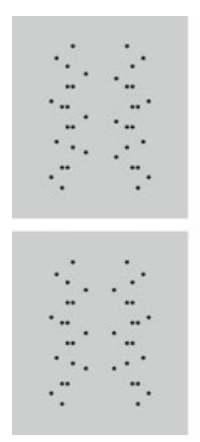

Far

Asymmetrical

Symmetrical

Symetrical

Asymmetrical
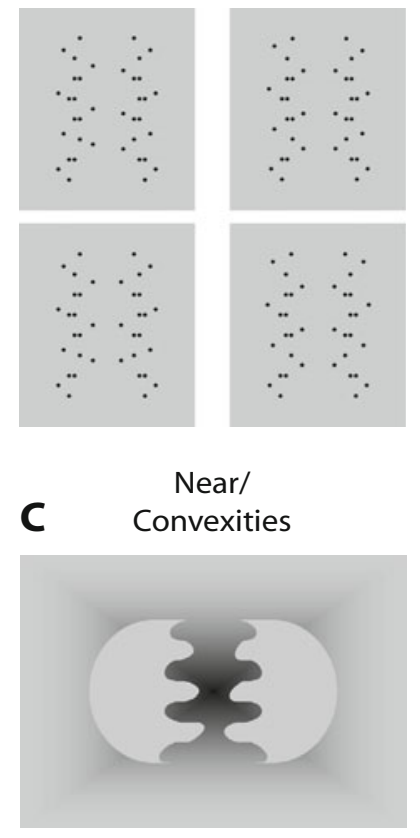

Symmetrical

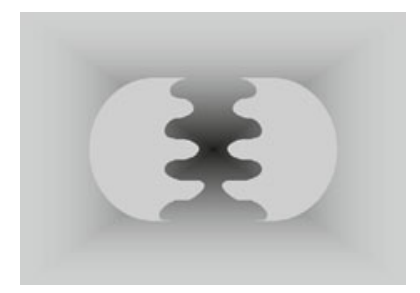

Near/

B Concavities

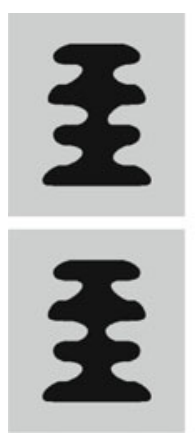

Far/

Convexities
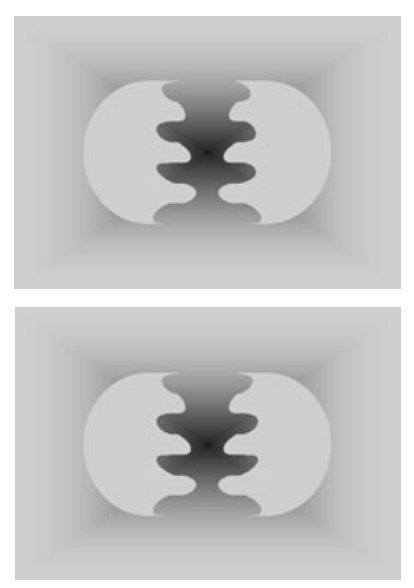

Figure 2. Examples of the stimuli used in our experiments. Top row, asymmetrical; bottom row, symmetrical. Left, perturbation near symmetry axis; right, perturbation far from symmetry axis. (A) Experiment 1. (B) Experiment 2. Concavities near symmetry axis, convexities far from axis. (C) Experiment 3. Convexities near symmetry axis, concavities far from axis. 
and far from axis (convexities) for Experiment 2, and near to axis (convexities) and far from axis (concavities) for Experiment 3.

\section{RESULTS}

The results of all three experiments are shown in Figure 3. In Experiment 1, we presented random dot patterns. The perturbations were either near or far from the axis of symmetry. From Figure 3, it is clear that perturbations near the axis were easier to detect than perturbations far from the axis, as was statistically confirmed using a paired, twotailed $t$ test $[.85$ vs. .50 correct; chance level $=.50 ; t(11)=$ 20.61, $p<.001]$.

In Experiment 2, we presented 2-D silhouettes. It is important to realize that the 2-D silhouettes were generated by exactly the same algorithm as the random dot patterns in Experiment 1. The only difference was that curves were fitted through the locations of the dots. Because of the way the curves were generated, we were able to selectively change the shape of the convexities without changing the shape of the concavities, and vice versa. As can be seen from Figure 3, the consequences of changing from random dots to silhouettes were dramatic. For the stimuli with perturbed concavities (near to axis), the proportion correct was .65, whereas for the perturbed convexities (far from axis) it was .75, again a significant difference $[t(19)=3.67, p<.002]$. Note that this difference goes in the opposite direction, in comparison with the equivalent near- and far-axis conditions of Experiment 1, resulting in a .45 swing in the proportions correct (a .2 decrease in the near-axis condition and a .25 increase in the far-axis condition relative to Experiment 1), and a significant experiment $\times$ type of perturbation interaction $[F(1,30)=127.2$, $p<.001]$, as revealed by a mixed-design ANOVA.
In Experiment 3, we again presented 2-D silhouettes. However, this time, we connected the dots in such a way that the result was a display containing two opposing silhouettes rather than a single silhouette. Consequently, the silhouettes in Experiment 3 were convex where the silhouettes in Experiment 2 were concave, and vice versa. As can be seen from Figure 3, this inversion of concave and convex yielded another inversion in the performance of the participants. Perturbations near the axis of symmetry now yielded .74 correct, whereas perturbations far from the axis of symmetry yielded .64 correct $[t(19)=3.24, p<.005]$. The inversion of convexities and concavities resulted in a .2 swing in proportions correct from Experiment 2 to Experiment 3 , as reflected in a significant experiment $\times$ type of perturbation interaction $[F(1,38)=23.55, p<.001]$.

\section{DISCUSSION}

Our results show that for 2-D silhouettes, it is easier to determine the symmetry of convexities than the symmetry of concavities. This convexity advantage is independent of the distance to the axis of symmetry. Central here are the results of Experiment 2: Even when the concavities are nearer the symmetry axis than the convexities, asymmetries in the convexities remain easier to detect. The random dot patterns in Experiment 1 are markedly different in this respect: Mismatches near the symmetry axis are considerably easier to detect. Finally, reversing the convexities and concavities in Experiment 3 reversed the pattern of results, demonstrating again that performance was determined by the shape descriptions, and not by the distance to the axis.

Our results offer further support for the idea that symmetry perception in 2-D shapes is primarily governed by

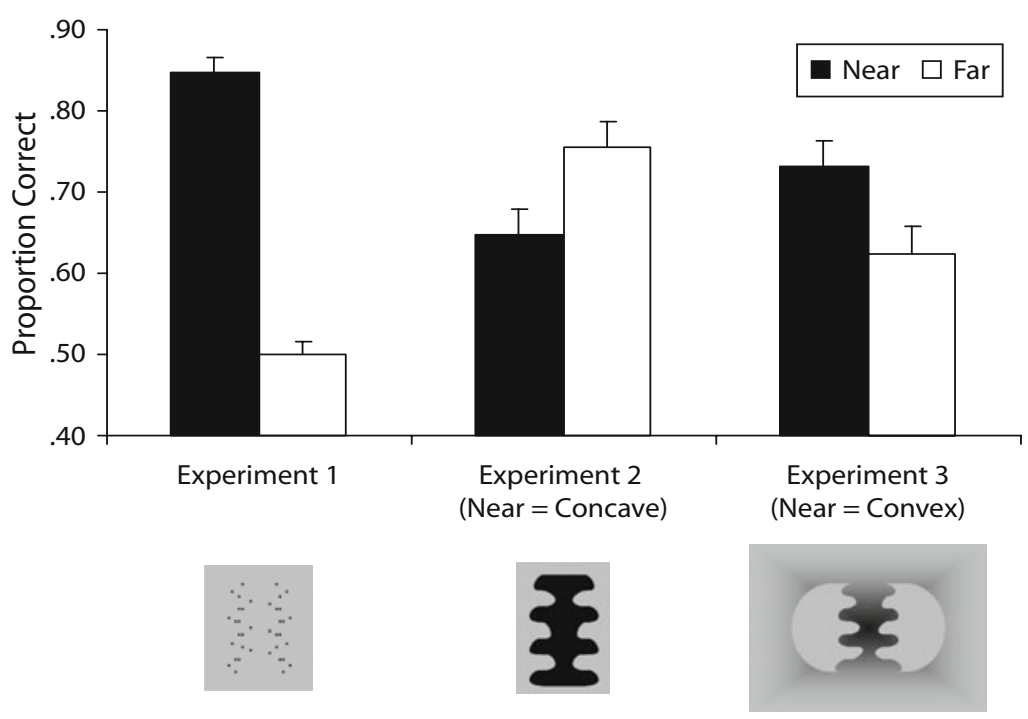

Figure 3. Results of the three experiments. Black bars, perturbation near symmetry axis; white bars, perturbation far from symmetry axis. In Experiment 2, the concavities were near the symmetry axis and the convexities far from the axis. In Experiment 3, the location of convexities and concavities was reversed. Error bars indicate within-subjects standard error. 
shape perception (i.e., shape perception precedes symmetry perception, even though theoretically, this would not have to be the case; Baylis \& Driver, 1995; Hulleman \& Humphreys, 2004). This shape perception is so strong that it even fully counteracts the normal bias toward the axis that exists in symmetry perception. We conclude that the shape descriptions of the figural convex areas are either more detailed than those of the figural concave areas, or are more effectively or efficiently utilized by symmetry detection processes. This conclusion holds even if the percept of symmetry (two objects) in Experiment 3 would be qualitatively different from the percept of symmetry (one object) in Experiment 2 (Baylis \& Driver, 2001). The definition of symmetry is equal in both experiments: The shape descriptions of both the convexities and the concavities have to match. Although the detection of symmetry might have been slower overall in Experiment 3 (note that we used longer presentation times), we would observe the result found only if the shape descriptions of concavities are poorer than those of convexities.

We only tested vertical symmetry. Of all orientations, vertical symmetry is the most salient bilateral symmetry (Wagemans, 1995), and therefore provides the strongest test of our hypotheses (as it would be most likely to involve early, fast, and parallel processes, generating the strongest bias toward the axis). There is therefore no a priori reason why our results would not extend to different orientations (e.g., horizontal), in which weaker symmetry-detection mechanisms may be overshadowed more by shape descriptions.

Several authors (e.g., Bertamini, 2001; Gibson, 1994) have shown that participants are faster in judging the relative positions of convex cusps than of concave cusps. This result has been explained as the consequence of the fact that the concave cusps are part boundaries, whereas the convex cusps correspond to the parts. Note, however, that in our experiments, the task was to match the shape of entire concavities, rather than just the positions of their curvature minima. Even if a part cut splits the concavity, a detailed description of the shape of the concavity on either side of the cut should contain sufficient information to judge whether the shapes of the concavities on either side of the symmetry axis are identical. Thus, our results go further than Gibson (1994) and Bertamini (2001), because they show that the entire shape description of a concavity, and not just the position of the cusp or the negative minimum inside it, is less detailed than that for convexity. This allows convexities to dominate the percept of symmetry. In other words, not just the part boundaries, but the entire concavities should be considered to be the "glue" between the convexities. In this respect, the results in Experiment 2 are especially revealing. Here, the concavities are nearest to the axis of symmetry (and to the point of fixation) and should therefore be represented at the highest resolution. But still, the participants were better at detecting irregularities in the convexities farther away from the axis of symmetry. This suggests that when serving shape perception, attention is directed to the convexities of a stimulus when the stimulus is presented at fixation.
Yet, on the other hand, there is also evidence that observers are more sensitive to shape changes in concavities than in convexities (Barenholtz et al., 2003; Cohen, Barenholtz, Singh \& Feldman, 2005). Furthermore, Hulleman et al. (2000) found that concavities attract attention, to the extent that they can be detected easily in visual search displays. This appears to contradict the supposed lack of interest the visual system has in concavities, as found here. However, it is important to make a distinction between the role concavities play in shape perception as part boundaries, and the shape of the concavities themselves. Concavities determine the part structure, and when a change in the shape of a concavity also changes the part structure of a shape, this will be particularly noticeable (Bertamini \& Farrant, 2005). But precisely because concavities are part boundaries, their actual shape is less important, and a shape change without a change in boundary (as was the case in our displays) will thus often go unnoticed. Consequently, concavities count for less in the perception of symmetry. There is a possibility that this conclusion actually might hold true for the perception of nonsymmetric shapes as well, as it has been suggested that the ease of symmetry detection reflects the use of symmetry in the representation of shape in general (see, e.g., Barlow \& Reeves, 1979; Burbeck \& Pizer, 1995; Wagemans, 1995).

\section{AUTHOR NOTE}

Correspondence regarding this article may be addressed to J. Hulleman, Department of Psychology, University of Hull, Cottingham Road, Hull HU6 7RX, England (e-mail: j.hulleman@hull.ac.uk) or C. N. L. Olivers, Department of Cognitive Psychology, Vrije Universiteit, Van der Boechorststraat 1, NL-1081 BT Amsterdam, The Netherlands (e-mail: cnl.olivers@psy.vu.nl).

\section{REFERENCES}

Barenholtz, E., Cohen, E. H., Feldman, J., \& Singh, M. (2003). Detection of change in shape: An advantage for concavities. Cognition, 89, 1-9.

Barlow, H. B., \& Reeves, B. C. (1979). The versatility and absolute efficiency of detecting mirror symmetry in random dot displays. Vision Research, 19, 783-793.

Baylis, G. C., \& Driver, J. (1994). Parallel computation of symmetry but not repetition within single visual shapes. Visual Cognition, 1, 377-400.

BAYLIS, G. C., \& Driver, J. (1995). Obligatory edge assignment in vision: The role of figure and part segmentation in symmetry detection. Journal of Experimental Psychology: Human Perception \& Performance, 21, 1323-1342.

Baylis, G. C., \& Driver, J. (2001). Perception of symmetry and repetition within and across visual shapes: Part-descriptions and objectbased attention. Visual Cognition, 8, 163-196.

Bertamini, M. (2001). The importance of being convex: An advantage for convexity when judging position. Perception, 30, 1295-1310.

Bertamini, M., \& FARRANT, T. (2005). Detection of change in shape: An advantage for changes in part structure. Acta Psychologica, 120, 35-54.

Burbeck, C. A, \& Pizer, S. M. (1995). Object representation by cores: Identifying and representing primitive spatial regions. Vision Research, 35, 1917-1930.

Cohen, E. H., Barenholtz, E., Singh, M., \& Feldman, J. (2005). What change detection tells us about the visual representation of shape. Journal of Vision, 5, 313-321.

Gibson, B. S. (1994). Visual attention and objects: One versus two or 
convex versus concave? Journal of Experimental Psychology: Human Perception \& Performance, 20, 203-207.

Hoffman, D. D., \& Richards, W. A. (1984). Parts of recognition. Cognition, 18, 65-96.

Hoffman, D. D., \& Singh, M. (1997). Salience of visual parts. Cognition, 63, 29-78.

Hulleman, J., \& Humphreys, G. W. (2004). Is there an assignment of top and bottom during symmetry perception? Perception, 33, 615-620.

Hulleman, J., Te Winkel, W., \& Boselie, F. (2000). Concavities as basic features in visual search: Evidence from search asymmetries. Perception \& Psychophysics, 62, 162-174.

Julesz, B. (1971). Foundations of Cyclopean perception. Chicago: University of Chicago Press.

Koenderink, J. J., \& VAn Doorn, A. J. (1982). The shape of smooth objects and the way contours end. Perception, 11, 129-137.
Olivers, C. N. L., \& VAN der Helm, P. A. (1998). Symmetry and selective attention: A dissociation between effortless perception and serial search. Perception \& Psychophysics, 60, 1101-1116.

Palmer, S. E. (1999). Vision science: Photons to phenomenology. Cambridge, MA: MIT Press.

Palmer, S. E., \& Hemenway, K. (1978). Orientation and symmetry: Effects of multiple, rotational, and near symmetries. Journal of Experimental Psychology: Human Perception \& Performance, 4, 691-702.

Wagemans, J. (1995). Detection of visual symmetries. Spatial Vision, 9, 9-32.

(Manuscript received August 31, 2006;

revision accepted for publication February 13, 2007.) 